\title{
Effects of risperidone and olanzapine on remnant-like lipoprotein particle cholesterol (RLP-C) in schizophrenic patients
}

\author{
Takahiko Nagamine \\ Division of Psychiatric Internal \\ Medicine, Seiwakai-Kitsunan Hospital, \\ Suzenji, Japan
}

Correspondence:Takahiko Nagamine Division of Psychiatric Internal Medicine, Seiwakai-Kitsunan Hospital, Suzenji 338I, Yamaguchi-shi, Yamaguchi-ken 747-1221, Japan

Email anagamine@ybb.ne.jp

\begin{abstract}
Second generation antipsychotics are associated with the risk of metabolic disorders such as diabetes mellitus and hyperlipidemia. Remnant-like lipoprotein particles cholesterol (RLP-C) are a known risk factor for cardiovascular events. The present study was performed to determine possible differences in fasting blood RLP-C levels between schizophrenic patients treated with risperidone as compared to olanzapine. Patients on olanzapine had significantly higher RLP-C levels than those on risperidone $(\mathrm{p}<0.01)$. In olanzapine-treated patients there was no abnormality in fasting blood glucose levels, but fasting insulin levels and homeostasis model assessment of insulin resistance (HOMA-IR) were elevated. RLP-C levels were significantly correlated with plasma triglyceride concentrations in both the olanzapine- $(p<0.01)$ and risperidone-treated patients $(\mathrm{p}<0.01)$. The regression line slope was greater for the olanzapine group, suggesting a greater influence of olanzapine on RLP-C. There was a significant correlation between RLP-C and HOMA-IR in the risperidone group $(\mathrm{p}<0.01)$ but not in the olanzapine group $(\mathrm{p}=0.80$ ). These results suggest that blood glucose monitoring may not be sufficient to detect metabolic disorder and that measurement of RLP-C might be helpful for the screening for metabolic disorders associated with olanzapine therapy.
\end{abstract}

Keywords: remnant-like lipoprotein particles cholesterol (RLP-C), schizophrenia, insulin resistance, risperidone, olanzapine

\section{Introduction}

The adverse reactions referred to as the metabolic syndrome (including diabetes mellitus and hyperlipidemia) associated with second generation antipsychotics have been gaining considerable attention. Approximately $40 \%$ of treated patients have the metabolic syndrome according to Clinical Antipsychotic Trials of Intervention Effectiveness (CATIE), a large-scale clinical study conducted to assess the usefulness of antipsychotic drugs (McEvoy et al 2005). The molecular mechanism that underlies the metabolic syndrome is insulin resistance and it has become common practice to monitor body weight and blood glucose levels in patients receiving second generation antipsychotics. However, the frequency of metabolic syndrome continues to increase and monitoring with more sensitive markers than blood sugar are required. Disorders of neutral fat metabolism are readily detectable and more sensitive than those of carbohydrate metabolism in patients with insulin resistance (Tanaka 2004). Of the neutral fats, the one which directly constitutes a risk for cardiovascular events is remnant-like lipoprotein particles cholesterol (RLP-C) (Devaraj et al 1998).

The present study was designed to determine whether there was any difference in blood RLP-C levels between treatment with risperidone and with olanzapine in 
patients in the maintenance stage of schizophrenia who had no indications of diabetes mellitus.

\section{Subjects and methods}

Outpatients with schizophrenia who initiated or were switched to either risperidone or olanzapine for at least 40 weeks were included in the study. Concomitant benzodiazepines were allowed. Inclusion criteria included a body mass index (BMI) of less than 30 and a fasting blood sugar level of less than $126 \mathrm{mg} / \mathrm{dL}$ prior to initiation of risperidone or olanzapine therapy. Patients whose dietary intake was unstable due to instability of disease condition and patients receiving treatment for diabetes mellitus or hyperlipidemia were excluded from the study. All patients fulfilling these criteria in our department from July 2002 to December 2004 and who gave their written informed consent were enrolled in the study.

Body weight measurement and collection of fasting blood samples were performed on any day after 40 weeks of treatment with oral risperidone or olanzapine. The following blood biochemical parameters were determined: RLP-C, total cholesterol, high-density lipoprotein (HDL) cholesterol, triglycerides, glucose, and insulin. RLP-C was determined in terms of cholesterol present in the remnant-like particles (RLP) fraction. RLP was measured as an unbound fraction in serum to anti-human apo A-I and anti-human apo B-100 monoclonal antibody immunoaffinity mixed gel (Nakajima et al 1996).

Differences between the olanzapine and risperidone groups were evaluated using unpaired, two-sided $t$-test, and any differences were considered statistically significant at $\mathrm{p}<0.05$. For each treatment group, the data were analyzed to see whether there is any significant correlation between RLP-C and triglycerides and between RLP-C and the homeostasis model assessment of insulin resistance (HOMA-IR).

\section{Results}

Age, study treatment duration and body weight changes

A total of 14 patients ( 8 males and 6 females) received risperidone and 14 patients (9 males and 5 females) received olanzapine. Patient demographic characteristics are shown in Table 1. No significant differences were noted between the two groups in terms of age, pre-treatment (baseline) body weight or treatment duration. The baseline blood sugar levels were significantly higher in the risperidone group.

Following treatment, small body weight changes were noted in both treatment groups compared to baseline values. Mean weight gain was $0.76 \mathrm{~kg}$ and $1.51 \mathrm{~kg}$ for the risperidone group and the olanzapine group, respectively. However, the intergroup difference in weight gain was not significant (Table 1).

\section{Metabolic indicators}

Post-treatment blood glucose levels were not significantly different between the two treatment groups. RLP-C values and serum triglyceride levels, however, were significantly higher in the olanzapine group (Table 2). There were no significant differences for total cholesterol and HDL cholesterol between the two treatment groups (Table 2). The olanzapine group showed significantly higher fasting blood insulin levels than the risperidone group and the HOMA-IR was also significantly higher for this group (Table 2).

\section{Relationship between RLP-C and triglycerides}

There was a significant correlation between the RLP-C levels and triglyceride levels in both the risperidone and olanzapine groups (Figure 1; $\mathrm{r}=0.8290, \mathrm{p}<0.001$ for the risperidone group; $r=0.8552, p<0.001$ for the olanzapine group). The slope of the regression line for the olanzapine group was

Table I Demographic characteristics

\begin{tabular}{llll}
\hline & $\begin{array}{l}\text { Risperidone-treated } \\
\text { patients }\end{array}$ & $\begin{array}{l}\text { Olanzapine-treated } \\
\text { patients }\end{array}$ & P value \\
\hline Age (years) & $57.5 \pm 7.2$ & $51.3 \pm 15.6$ & 0.19 \\
Sex & $6 \mathrm{f} / 8 \mathrm{~m}$ & $5 \mathrm{f} / 9 \mathrm{~m}$ & - \\
Baseline body weight $(\mathrm{kg})$ & $59.7 \pm 8.5$ & $64.6 \pm 11.9$ & 0.23 \\
Baseline glucose $(\mathrm{mg} / \mathrm{dL})$ & $116.2 \pm 9.0$ & $101.7 \pm 6.8$ & $<\mathbf{0 . 0 1}$ \\
Treatment duration (weeks) & $135.2 \pm 46.8$ & $101.1 \pm 43.8$ & 0.057 \\
Mean antipsychotic dose $(\mathrm{mg} /$ day) & $4.7 \pm 2.0$ & $13.2 \pm 4.6$ & - \\
Antipsychotic dose range & $2-8 \mathrm{mg}$ & $5-20 \mathrm{mg}$ & - \\
Body weight change during treatment $(\mathrm{kg})$ & $0.76 \pm 1.30$ & $1.51 \pm 1.89$ & 0.23 \\
\hline
\end{tabular}

Notes: Values are given as mean \pm SEM. Significant $p$ values are indicated in bold text. 
Table 2 Post-treatment carbohydrate and lipid metabolism markers

\begin{tabular}{llll}
\hline & $\begin{array}{l}\text { Risperidone-treated } \\
\text { patients }\end{array}$ & $\begin{array}{l}\text { Olanzapine-treated } \\
\text { patients }\end{array}$ & P value \\
\hline Glucose $(\mathrm{mg} / \mathrm{dL})$ & $119.1 \pm 13.3$ & $1 \mathrm{II.9 \pm 16.6}$ & 0.22 \\
Insulin $(\mu / \mathrm{L} / \mathrm{L})$ & $5.5 \pm 3.1$ & $9.2 \pm 4.2$ & $\mathbf{0 . 0 1 5}$ \\
HOMA-IR & $1.65 \pm 0.95$ & $2.50 \pm 1.13$ & $\mathbf{0 . 0 4 2}$ \\
RLP cholesterol $(\mathrm{mg} / \mathrm{dL})$ & $4.17 \pm 1.77$ & $9.80 \pm 5.32$ & $<\mathbf{0 . 0 I}$ \\
Triglycerides $(\mathrm{mg} / \mathrm{dL})$ & $105.6 \pm 62.3$ & $185.9 \pm 87.9$ & $<\mathbf{0 . 0 I}$ \\
Total cholesterol $(\mathrm{mg} / \mathrm{dL})$ & $196.1 \pm 27.9$ & $189.1 \pm 32.5$ & 0.55 \\
HDL cholesterol $(\mathrm{mg} / \mathrm{dL})$ & $61.7 \pm 11.9$ & $57.4 \pm 16.8$ & 0.44 \\
\hline
\end{tabular}

Notes: Values are given as mean \pm SEM. Significant $p$ values are indicated in bold text.

Abbreviations: HOMA-IR, homeostasis model assessment of insulin resistance; RLP, remnant-like lipoprotein particles; HDL, high density lipoprotein.

about two-fold greater than that of the regression line for the risperidone group.

\section{Relationship between RLP-C and HOMA-IR}

There was also a significant correlation of RLP-C with HOMA-IR in the risperidone group (Figure 2; $\mathrm{r}=0.7323$, $\mathrm{p}=0.003$ ); whereas in the olanzapine group, there was no such relationship (Figure 2; $\mathrm{r}=0.0739, \mathrm{p}=0.80$ ).

\section{Discussion}

Blood RLP-C levels were measured in patients with stable chronic phase schizophrenia receiving risperidone or olanzapine therapy in the usual outpatient setting of a clinical practice. The observation period was approximately two years (mean 135.2 weeks for the risperidone group and 101.1 weeks for the olanzapine group). Both therapy groups showed only minimal body weight changes during this period. The baseline (pre-treatment) blood sugar levels were significantly higher in the risperidone group. This difference may be due to the fact that olanzapine, which is contraindicated in diabetic patients in Japan, is unlikely to be prescribed to patients with blood glucose levels at the upper level of the normal range. Since RLP-C levels are not routinely measured in clinical practice in Japan, baseline values (before antipsychotic therapy) were not available. We have assumed

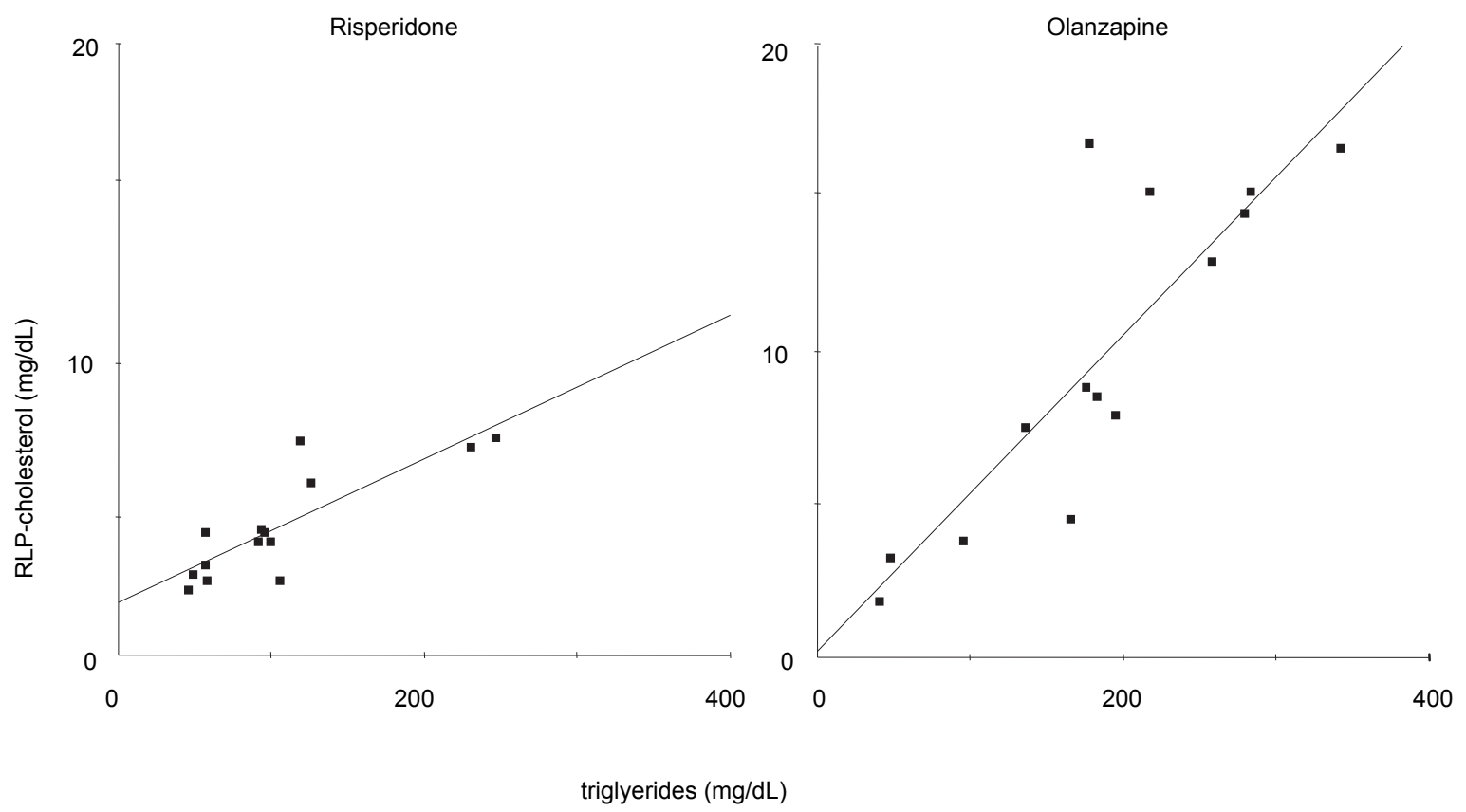

Figure I Relationship between levels of RLP-C and triglycerides.

Risperidone $r=0.829042 p=0.0002$.

Olanzapine $r=0.855195 p<0.0001$. 


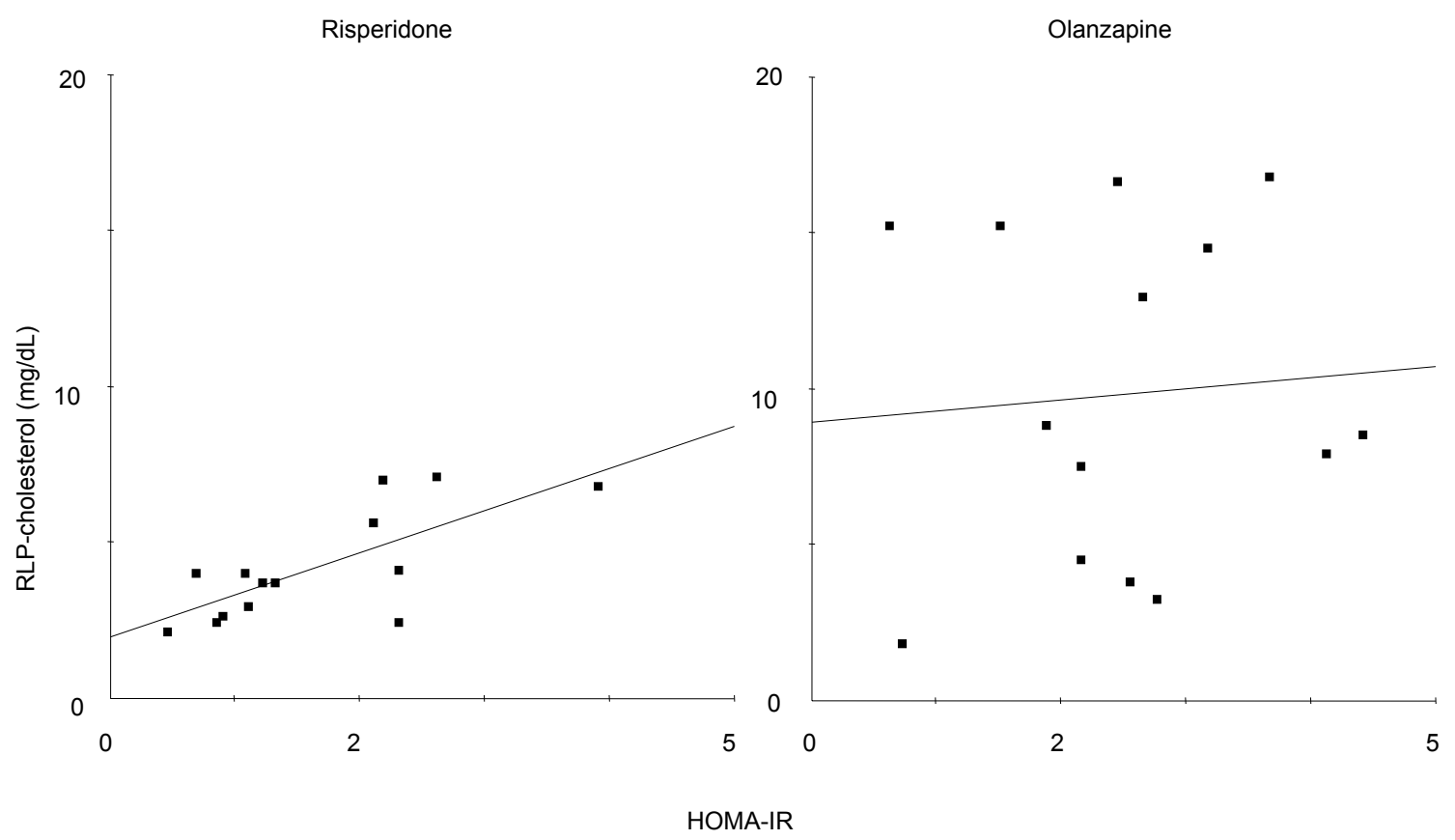

Figure 2 Relationship between levels of RLP-C and HOMA-IR.

Risperidone $r=0.732270 p=0.0029$.

Olanzapine $r=0.073893 p=0.8018$

that the two groups would have had comparable baseline levels RLP-C levels. It is however, possible that since patients treated with risperidone had higher baseline glucose levels, they may also have had higher RLP-C levels. Higher glucose levels and higher RLP-C levels have been shown tend to go together in diabetic patients (Tanaka 2004). In this case the difference seen in RLP-C levels following therapy would be even more meaningful.

Following treatment, blood glucose, total cholesterol, and HDL cholesterol were within normal limits in both groups and showed no significant intergroup difference. Blood RLP-C levels were within the normal limits in the risperidone group but in the olanzapine group they were significantly higher. The olanzapine group showed normal blood glucose levels with elevated fasting blood insulin values. The insulin resistance indicator HOMA-IR for this group (mean: 2.50) exceeded the reference value of 2 and differed significantly from the value found for the risperidone group (mean: 1.65) which was within normal limits.

Results of large-scale clinical trials (CATIE) have indicated the therapeutic usefulness of risperidone and olanzapine, which were randomized to patients who had dropped out of previous drug therapies. The dropout rates with risperidone and olanzapine were lower than with quetiapine and ziprasidone (Stroup et al 2005). However, when viewed in terms of metabolism and triglycerides in particular, a significant elevation in triglycerides was observed in patients receiving olanzapine, whereas this metabolic indicator was decreased in patients receiving risperidone even after adjustment for the duration of exposure to the drugs (Akuzawa et al 1995).

In the present study, the olanzapine group also exhibited significantly higher serum triglyceride levels than the risperidone group. RLP-C is an intermediate metabolite of triglycerides. Under normal conditions, RLP-C is metabolized very rapidly. Lipoprotein lipase activity, hepatic triglylipase activity, and remnant receptor activity are involved in the catabolism of RLP-C. All these enzyme and receptor activities are diminished in the presence of insulin resistance. The elevated RLP-C seen in the olanzapine group in the present study is probably the consequence of the insulin resistance as indicated by the rise in HOMA-IR.

RLP-C is readily taken up by macrophages in the arterial wall and facilitates foamy changes of the macrophages, thereby leading to formation of atherosclerotic plaques. RLP-C has been demonstrated to promote arteriosclerosis via formation of atherosclerotic plaques due to enhanced uptake by macrophages, increased platelet aggregation, enhanced PAI-1 activity, impaired vascular endothelial cell function, and enhanced monocyte adhesion to the endothelium (Tanaka et al 2004). RLP-C may be elevated in patients with coronary 
diseases, has been described to correlate with pathological changes in coronary arteries, and is increasingly seen as a risk factor for cardiovascular events (Takeichi et al 1999).

Although insulin resistance is presumed to be the cause of the high RLP-C levels in patients receiving olanzapine in this study, neither treatment group showed appreciable body weight changes ( $<7 \%$ in both groups). The insulin resistance observed in the olanzapine group might thus be attributable to a difference between olanzapine and risperidone, namely, a possible direct effect of olanzapine to induce insulin resistance, rather an effect associated with body weight gain. The mechanism whereby olanzapine exerts a direct effect on metabolism remains unclear. Nonetheless, several previous studies have shown olanzapine to be directly linked to insulin resistance. Based on assessment of HOMA-IR and an oral glucose tolerance test, clozapine and olanzapine, but not risperidone and other atypical antipsychotic drugs, have been shown to cause insulin resistance in a patient population adjusted for BMI to eliminate the effects of weight gain (Newcomer et al 2002). A study in non-obese, non-diabetic patients showed that patients receiving clozapine or olanzapine had high fasting blood insulin levels and increased HOMA-IR and leptin values, again indicating insulin resistance (Henderson et al 2005). They also showed that the insulin sensitivity index calculated by minimal model analysis, based on an intravenous glucose tolerance test (IVGTT), was significantly lower for clozapine and olanzapine, compared to risperidone (Henderson et al 2005).

The relationship of RLP-C with triglycerides differed slightly between the risperidone group and the olanzapine group. In both groups, RLP-C was significantly correlated with triglycerides (Figure 1). The regression equation for the risperidone group in this study closely resembles that of correlation between RLP-C and triglycerides for healthy Japanese adult volunteers (Akuzawa et al 1995) which was considered to represent a physiological correlation. However, the slope of the regression line for the olanzapine group was about two-fold greater than that of the risperidone group. This suggests that patients with increased triglyceride levels might show a further elevation in RLP-C during olanzapine therapy suggesting that RLP-C metabolism maybe slowed in the olanzapine-treated patients. It would be reasonable to assume that the difference concerning the correlation between the intermediate of neutral fat RLP-C and triglycerides indicates a qualitative change in triglyceride-rich lipoprotein. RLP-C are heterogenous in their physical and biochemical properties because they are assayed by immunoseparation. In subjects with increased serum triglycerides, the ratio of
RLP-C to remnant-like particles triglyceride (RLP-TG) correlates with the particle size, an important factor in the atherogenic effect, while the ratio of RLP-C to triglycerides does not correlate with particle size (Okazaki et al 2002). The increase in RLP-C has been shown to be inversely proportional to LDL cholesterol particle size, so that elevation in RLP-C leads to an increase in small dense LDL which promotes progression of atherosclerosis (Takayanagi et al 2004). Enhanced RLP-C is related to an increase in atherogenic smaller-sized lipoprotein (Takayanagi et al 2004). The difference in pattern of correlation between RLP-C and triglycerides (the slope of the regression line) observed between the olanzapine group and the risperidone group suggests that the particle size of lipoproteins may differ between the two groups. With the aim of estimating the atherogenicity it would be of interest, in the future, to evaluate triglycerides in the context of the RLP-C lipoprotein, just as cholesterol is evaluated in terms of the LDL-C and HDL-C lipoproteins.

The present data obtained on the relationship between RLP-C and HOMA-IR show completely different features between for the two therapy groups with a significant correlation of RLP-C with HOMA-IR only in the risperidone group (Figure 2). A significant positive correlation between HOMA-IR and RLP-C has been reported in the general Japanese population (Ohnishi et al 2002). In contrast, in patients receiving olanzapine, RLP-C did not correlate with HOMA-IR (Figure 2). The olanzapine group displayed blood glucose levels within the normal limits but elevated RLP-C and insulin levels and no correlation of RLP-C to HOMAIR. These results suggest that, in the patients with chronic schizophrenia undergoing long-term olanzapine therapy, RLP-C may be in a state where it can no longer be controlled with insulin.

The weaknesses of the present study are principally its relatively small size and the fact that the patients were not randomized to one treatment or another. A number of factors can affect development of diabetes such as race, family history of diabetes, life-style, etc. Subjects from the two groups could not therefore be matched precisely. In addition all the subjects were of Japanese descent and it remains to be shown to what extent these results can be extended to other races. A larger randomized controlled trial is necessary to corroborate these data.

\section{Conclusion}

Patients with chronic schizophrenia showing stable blood glucose levels are generally considered not to have any metabolic disorder. In patients receiving olanzapine, however, RLP-C, 
an important risk factor for development of cardiovascular events, may be elevated even though their blood glucose levels are within the normal limits. Patients treated with risperidone showed relationships between RLP-C and triglycerides and between RLP-C and HOMA-IR which were comparable with those seen in the general population, whereas patients taking olanzapine appeared to be in a state where RLP-C might not be controlled with insulin.

For insulin resistance, RLP-C is a more sensitive marker than blood glucose. Early detection of insulin resistance cannot be achieved by blood sugar monitoring alone. It is proposed that in patients taking olanzapine it may be helpful to monitor for metabolic disorders by measuring plasma $\mathrm{RLP}-\mathrm{C}$ to detect insulin resistance even if the blood glucose level is normal.

\section{References}

Akuzawa K, Nakano T, Sekiguchi I, et al. 1995. Remnant-like particlescholesterol assay and its clinical application to lipid tests in a postprandial as well as fasting state. Jpn J Clin Pathol, 43:1159-67.

Devaraj S, VagaG, Lange R, et al. 1998. Remnant-like particle cholesterol levels in patients with dysbetalipoproteinemia or coronary artery disease. Am J Med, 104:445-50.
Henderson DC, Cagliero E, Copeland PM, et al. 2005. Glucose metabolism in patients with schizophrenia treated with atypical antipsychotic agents. Arch Gen Psychiatry, 62:19-28.

McEvoy JP, Meyer JM, Goff DC, et al. 2005. Prevalence of the metabolic syndrome in patients with schizophrenia. Schizophr Res, 80:19-32.

Nakajima K, Okazaki M, Tanaka A, et al. 1996. Separation and determination of remnant-like particles in human serum using monoclonal antibodies to apoB100 and apoA-I. J Clin Ligand Assay, 19:177-83.

Newcomer JW, Haupt DW, Fucetola R, et al. 2002. Abnormalities in glucose regulation during antipsychotic treatment of schizophrenia. Arch Gen Psychiatry, 59:337-45.

Ohnishi H, Saitoh S, Takagi S, et al. 2002. Relationship between insulinresistance and remnant-like particle cholesterol. Atherosclerosis, 164:167-70.

Okazaki M, Usui S, Tada N, et al. 2000. Relationship between RLPtriglyceride to RLP-cholesterol ratio and particle size distribution in RLP-cholesterol profiles by HPLC. Clin Chim Acta, 296:135-49.

Stroup TS, Lieberman JA, McEvoy JP, et al. 2006. Effectiveness of olanzapine, quetiapine, risperidone, and ziprasidone in patients with chronic schizophrenia following discontinuation of a previous atypical antipsychotic. Am J Psychiatry, 163:611-22.

Takayanagi N, Onuma T, Kato S, et al. 2004. Association between LDL particle size and postprandial increase of remnant-like particles in Japanese type 2 diabetic patients. Diabetes Res Clin Pract, 66:245-52.

Takeichi S, Yukawa N, Nakajima Y, et al. 1999. Association of plasma triglyceride-rich lipoprotein remnants with coronary atherosclerosis in cases of sudden cardiac death. Atherosclerosis, 142:309-15.

Tanaka A. 2004. Postprandial hyperlipidemia and atherosclerosis. J Atherosclerosis Thrombosis, 11:322-9. 